http://jmscr.igmpublication.org/home/ ISSN (e)-2347-176x ISSN (p) 2455-0450

crossref DOI: https://dx.doi.org/10.18535/jmscr/v7i12.65

\title{
Retrospective Comparative Study between Capecitabine and Gemcitabine Based Chemotherapy in the Treatment of Locally Advanced Unresectable Carcinoma of Gall Bladder
}

\author{
Authors \\ Dr Dharmaraj. A ${ }^{1}$, Dr SN Prasad ${ }^{2}$, Dr Tazyeen Fatima ${ }^{3}$, Dr Jitendra Kumar Verma ${ }^{4}$, \\ Dr Pramod Kumar Singh ${ }^{5}$, Dr Archana Singh ${ }^{6}$, Dr Mohd Waseem Raza ${ }^{7}$ \\ 1,3 Junior Resident JK Cancer Institute Kanpur \\ ${ }^{2}$ HOD \& Professor JK Cancer Institute \\ ${ }^{4,5,6,7}$ Assistant Professor JK Cancer Institute
}

\begin{abstract}
Introduction: Locally advanced carcinoma of gall bladder is a disease with a dismal prognosis. Chemotherapy is the main stay of treatment, but there is no standard chemotherapy options for patients with GB carcinoma

Aim: To compare the overall survival, time to disease progression and toxicity between the two chemotherapeutic regimens

Materials and Methods: Single institution retrospective study in 2018 of patients treated with capecitabine and gemcitabine based chemotherapy-gemcitabine either with cisplatin or carboplatin. A total of 230 patients were identified, of whom 67 patients were treated with chemotherapy,34 received capecitabine and 33 patients received gemcitabine either with cisplatin or carboplatin

Results: We observed the overall survival and time to disease progression were similar in outcomes in both regimens. No major acute toxicities seen in both arms

Conclusion: Gemcitabine based chemotherapy provides similar outcomes to capecitabine, but capecitabine also offers the advantage of oral dosing thus facilitating drug delivery and patient compliance

Keywords: gemcitabine, capecitabine, locally advanced gall bladder carcinoma.
\end{abstract}

\section{Introduction}

Gall bladder carcinoma although less common than other malignancies, patients often present with locally advanced or metastatic disease which is the reflection of their aggressive biology, late stage at diagnosis and poor prognosis with an average 5 year survival rate of $5 \%$.

Previous phase II study using gemcitabine and capecitabine showing median time to tumor progression of 9 months and median overall survival of 14 months but regimen is toxic with grade $3 / 4$ hematological toxicities.

None of study has been done to compare efficacy between gemcitabine and capecitabine. The purpose of our study is therefore to provide an updated analysis of survival outcomes and toxicities between two arms. 


\section{Materials and Methods}

In our study, 67 previously untreated patients with histopathologically proven locally advanced inoperable gall bladder carcinoma were included from January $1^{\text {st }}$ to December $31^{\text {st }} 2018$.

In one arm ,patients were treated with gemcitabine at the dose of $1000 \mathrm{mg} / \mathrm{m} 2$ iv day 1 and either with cisplatin $75 \mathrm{mg} / \mathrm{m} 2$ or carboplatin AUC 5 iv day 1 repeated every 21 days and in other arm, capecitabine is administered orally at $825 \mathrm{mg} / \mathrm{m} 2$ BD daily for 14 days every 3 weekly.

Overall survival was defined as the time from starting of treatment until death or last follow up. All patients were evaluated for toxicity from the time of their first day of treatment.

\section{Results}

Overall the number of female patients is much higher than male patients. Median age was $55 \mathrm{yrs}$ (ranges from 40-65). Most of the patients had KPS 70. Most common presenting complaint was pain followed by loss of appetite. Commonly observed histopathology was adenocarcinoma.

\begin{tabular}{|l|c|c|}
\hline & $\begin{array}{c}\text { Gemcitabine } \\
\text { Arm }\end{array}$ & $\begin{array}{c}\text { Capecitabine } \\
\text { Arm }\end{array}$ \\
\hline Overall Survival & 4 Months & 4 Months \\
\hline Time to Disease Progression & 3 Months & 4 Months \\
\hline
\end{tabular}

None of the patients experienced major acute toxicities that required hospitalization after chemotherapy in both arms.

\section{Discussion}

Several studies have shown the benefit of gem-cap regimen for advanced gall bladder carcinoma but none of the studies reported the comparison between them. As a retrospective study, there is potential for incomplete capture of all patients with advanced GB carcinoma. Missing data and recall bias are also potential short comings of this study.

Direct comparison of this retrospective study with previous prospective trials is challenging as the differences in methodology limit and comparative statistical analysis. Therefore comparison of this study with others can only be performed in a historical fashion.

Similar survival outcomes and favourable toxicity were observed in this analysis in both arms and are consistent with other studies.

\section{Conclusion}

Both regimens provides similar outcomes in overall survival and in time to disease progression but in terms of patient compliance and drug delivery capecitabine offers little advantage over gemcitabine based chemotherapy. More prospective comparison of these regimens are therefore warranted. 